\title{
Elliptic Blending with One-Equation Model
}

\author{
By M. M. Rahman, Xiaoqing Tian, Huachen Pan \& A. K. M. Sadrul Islam
}

Abstract- A wall-distance-free modification to Wray-Agarwal (WA) one-equation turbulence model is convoked using an elliptic relaxation approach to accurately accounting for non-local characteristics of near-wall turbulence. Model coefficients/functions are parameterized with the elliptic relaxation function to preserve the combined effects of near-wall turbulence and nonequilibrium. The characteristic length scale associated with the elliptic relaxation equation is formulated in terms of viscous and turbulent length scales in conjunction with the invariant of strain-rate tensor. Consequently, non-local effects are explicitly influenced by the mean flow and turbulent variables. A near-wall damping function is introduced to relax the viscous length-scale coefficient adhering to the elliptic relaxation model. Comparisons indicate that the new model improves the accuracy of flow simulations compared to the widely used Spalart-Allmaras model and remains competitive with the SST $k$ - $\omega$ model. A good correlation is obtained between the current model and DNS/experimental data.

Keywords: damping function; wall-distance-free; elliptic blending; non-equilibrium flow.

\section{GJSFR-F Classification: MSC 2010: 11G05}

Strictly as per the compliance and regulations of:

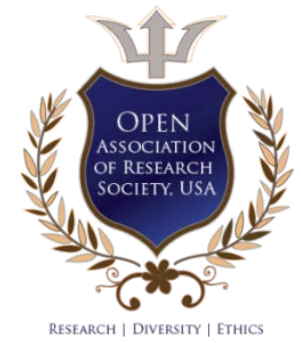

(C) 2020. M. M. Rahman, Xiaoqing Tian, Huachen Pan \& A. K. M. Sadrul Islam. This is a research/review paper, distributed under the terms of the Creative Commons Attribution-Noncommercial 3.0 Unported License http://creativecommons.org/licenses/by$\mathrm{nc} / 3.0 /$ ), permitting all non commercial use, distribution, and reproduction in any medium, provided the original work is properly cited. 

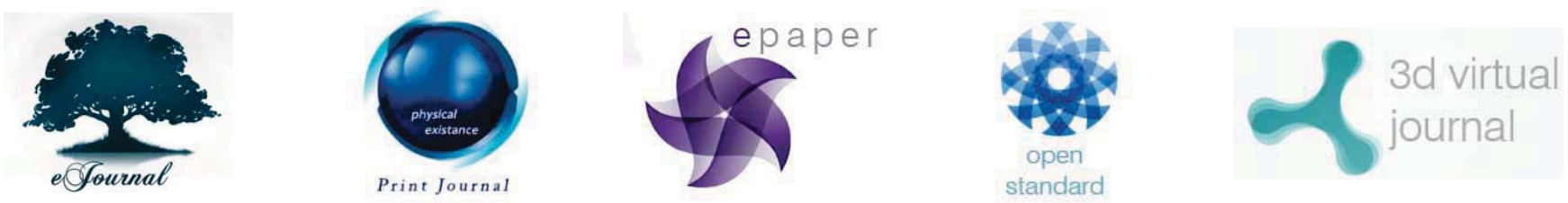

\title{
Elliptic Blending with One-Equation Model
}

\author{
M. M. Rahman ${ }^{\alpha}$, Xiaoqing $\operatorname{Tian}^{\sigma}$, Huachen Pan ${ }^{\circ} \&$ A. K. M. Sadrul Islam ${ }^{\omega}$
}

Abstract- A wall-distance-free modification to Wray-Agarwal (WA) one-equation turbulence model is convoked using an elliptic relaxation approach to accurately accounting for non-local characteristics of near-wall turbulence. Model coefficients/functions are parameterized with the elliptic relaxation function to preserve the combined effects of nearwall turbulence and nonequilibrium. The characteristic length scale associated with the elliptic relaxation equation is formulated in terms of viscous and turbulent length scales in conjunction with the invariant of strain-rate tensor. Consequently, non-local effects are explicitly influenced by the mean flow and turbulent variables. A near-wall damping function is introduced to relax the viscous length-scale coefficient adhering to the elliptic relaxation model. Comparisons indicate that the new model improves the accuracy of flow simulations compared to the widely used Spalart-Allmaras model and remains competitive with the SST $k$ - $\omega$ model. A good correlation is obtained between the current model and DNS/experimental data.

Keywords: damping function; wall-distance-free; elliptic blending; non-equilibrium flow.

\section{Nomenclature}

$C_{1} \quad$ model closure coefficient

$C_{b} \quad$ model closure coefficient

$C_{w}$ model closure coefficient

$C_{f} \quad$ skin-friction coefficient

$C_{1 k \epsilon}$ closure coefficient

$C_{2 k \omega}$ closure coefficients

$C_{2 k \epsilon}$ closure coefficient

$d \quad$ distance to the nearest wall

$W$ mean vorticity invariant

$u^{+} \quad$ non-dimensional flow velocity

$y^{+} \quad$ non-dimensional wall distance $f_{\mu} \quad$ eddy-viscosity damping function

$f_{1}$ blending function

$f_{R}$ elliptic blending function

$h$ channel height

$k$ turbulent kinetic energy

$R$ undamped eddy-viscosity; $k / \omega$

Re Reynolds number

$S$ mean strain-rate invariant

$\chi$ undamped turbulent-to-laminar viscosity ratio

$\omega$ specific turbulent dissipation rate

Subscript

$\mu, \mu_{T}$ laminar and turbulent eddy viscosities $\mathrm{T}$ turbulent condition

$\nu \quad$ kinematic viscosity $\quad \mathrm{i}, \mathrm{j}$ variable numbers

$\rho$ density

ref reference condition

$\sigma \quad$ Schmidt number

\section{INTRODUCTION}

One-equation turbulence models solve directly for the eddy-viscosity $\left(\mu_{T}\right)$ without computing the full range of turbulent time and length scales. This form of transport equation is attrac-

\footnotetext{
Author a $\sigma$ p: Hangzhou Dianzi University, School of Mechanical Engineering, 310018 Hangzhou, China. e-mails: mizanur_rahman@hdu.edu.cn,md.mizan68@gmail.com

Author w: X Professor, BUET, Department of Mechanical Engineering, Dhaka-1000, Bangladesh.
} 
tive due to its simplicity of implementation and less demanding computational requirements, compared with the standard two-equation $k-\epsilon$ and $k-\omega$ models. However, several one-equation eddy-viscosity $\left(\mu_{T}\right)$ turbulence models are unable to accurately predict non-equilibrium flows, e.g., wall-bounded flows in adverse pressure gradient with separation and reattachment. This can be largely attributed to the overestimation of $\mu_{T}$ by the model. Thus, the inclusion of an elliptic blending in the $\mu_{T}$-transport equation may improve the prediction capability of oneequation model.

In particular, many turbulence models usually include the distance to the wall as an explicit parameter which hinders them from simulating complex flows involving multiple surfaces; the wall distance in this case becomes cumbersome to be defined accurately. The elliptic relaxation method is an excellent way to avoid the use of wall-distance in an eddy-viscosity turbulence model [1-10]. The wall blocking is governed by an elliptic partial differential equation (i.e., the Helmholtz-type equation) which accounts for non-local near-wall effects. Durbin et al. [7], Rahman et al. [8,9] and Elkhoury [10] have employed a Helmholtz-type relaxation equation along with a one-equation model to account for the wall-blocking effect.

Recently, a new one-equation model, the Wray-Agarwal (WA) model based on $R=k / \omega$ $[11,12]$ has been proposed which has shown promise for accurately predicting many wallbounded mildly separated flows. However, the model contains explicitly the distance from the wall. This paper proposes wall-distance-free (wdf) modifications to the WA model with an elliptic blending function. Nevertheless, the damping function is retained to relax the viscous length-scale coefficient embedded with the elliptic relaxation equation. The elliptic WA (EWA) model is applied to compute the fully-developed turbulent flow in a channel, the turbulent flow in an asymmetric planar diffuser and the flow over an Onera-M6 wing. Computations show that the EWA model improves the predictions compared to the original WA (OWA) model.

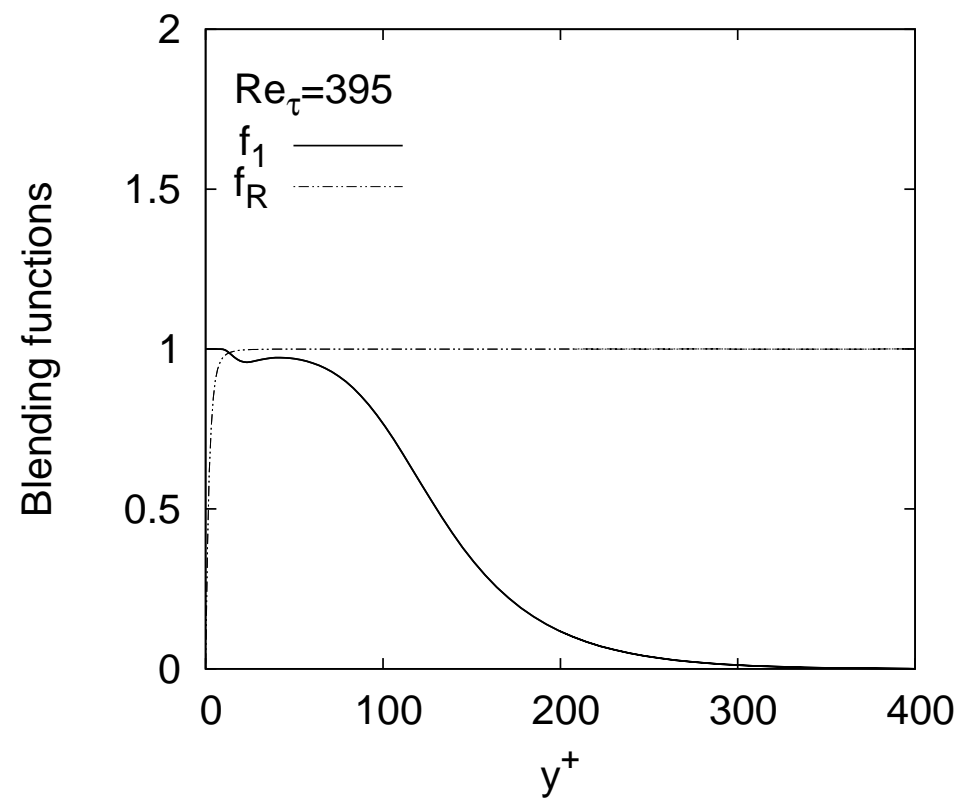

Figure 1: Blending function distributions for turbulent channel flow

III. Original WA(OWA) Model

The OWA model determines the undamped eddy-viscosity $R=k / \omega$ by the following transport equation $[11,12]$ : 


$$
\begin{aligned}
\frac{\partial \rho R}{\partial t}+\frac{\partial \rho U_{j} R}{\partial x_{j}} & =\frac{\partial}{\partial x_{j}}\left[\left(\mu+\sigma_{R} \mu_{T}\right) \frac{\partial R}{\partial x_{j}}\right]+C_{1} \rho R S+\rho f_{1} C_{2 k \omega} \frac{R}{S} \frac{\partial R}{\partial x_{j}} \frac{\partial S}{\partial x_{j}} \\
& -\rho\left(1-f_{1}\right)\left[C_{2 k \epsilon}\left(\frac{R}{S}\right)^{2} \frac{\partial S}{\partial x_{j}} \frac{\partial S}{\partial x_{j}}\right]
\end{aligned}
$$

Ref

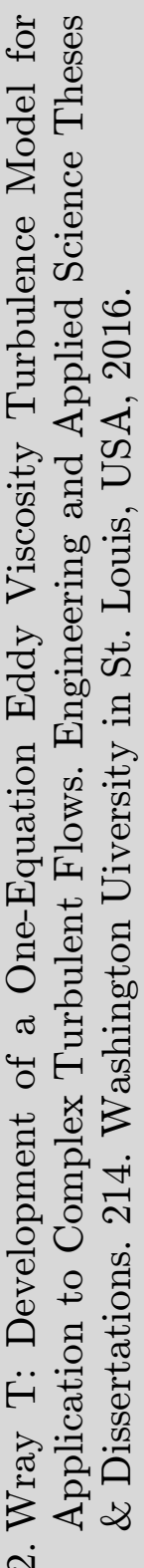

ำ

where the eddy viscosity $\mu_{T}=f_{\mu} \rho R$ and other variables are evaluated as:

$$
\begin{aligned}
f_{1} & =\tanh \left(\arg _{1}^{4}\right), & \arg _{1} & =C_{b} \frac{\nu+R}{S \kappa^{2} d^{2}} \\
f_{\mu} & =\frac{\chi^{3}}{\chi^{3}+C_{w}^{3}}, & \chi & =\frac{R}{\nu} \\
C_{1} & =f_{1}\left(C_{1 k \omega}-C_{1 k \epsilon}\right)+C_{1 k \epsilon}, & \sigma_{R} & =f_{1}\left(\sigma_{k \omega}-\sigma_{k \epsilon}\right)+\sigma_{k \epsilon}
\end{aligned}
$$

where $d$ is the normal distance from the wall and $\nu$ represents the kinematic viscosity. The associated constants are [12]: $C_{1 k \omega}=0.0833, C_{1 k \epsilon}=0.16, C_{2 k \omega}=1.22, C_{2 k \epsilon}=1.95$, $\sigma_{k \omega}=0.72, \sigma_{k \epsilon}=1.0, C_{b}=1.66, C_{w}=8.54$ and $\kappa=0.41$. The in-variants of mean strain-rate and vorticity tensors are given by $S=\sqrt{2 S_{i j} S_{i j}}$ and $W=\sqrt{2 W_{i j} W_{i j}}$ (required afterwards), respectively. The strain-rate $S_{i j}$ and the vorticity $W_{i j}$ tensors are defined as

$$
S_{i j}=\frac{1}{2}\left(\frac{\partial u_{i}}{\partial x_{j}}+\frac{\partial u_{j}}{\partial x_{i}}\right), \quad W_{i j}=\frac{1}{2}\left(\frac{\partial u_{i}}{\partial x_{j}}-\frac{\partial u_{j}}{\partial x_{i}}\right)
$$

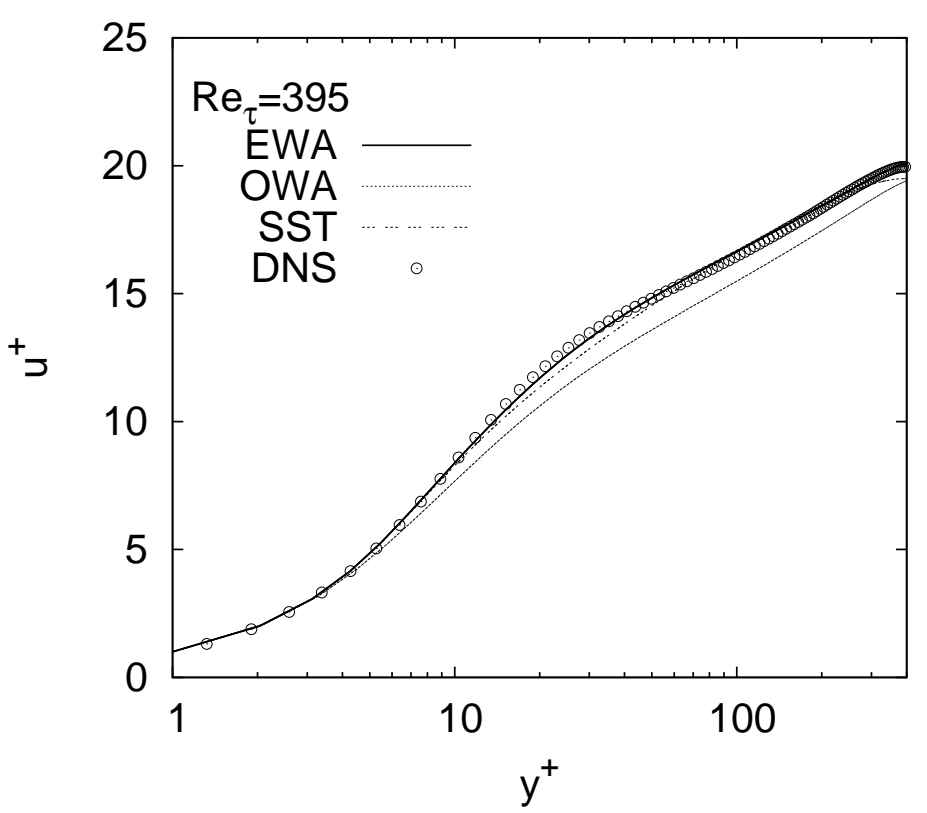

Figure 2: Channel flow predictions compared with DNS results

A plot of blending function related to the OWA model in Eq. (2) for a fully-developed channel flow with $R e_{\tau}=395$ is shown in Fig. 1 . As can be seen, for the OWA model $f_{1}=1.0$ in the viscous sub-layer and in the logarithmic overlap region; finally approaches 0 far away from the wall. 


\section{Elliptic WA (EWA) Model}

To assess the impact of elliptic relaxation function to account for non-local wall effects and flow in-homogeneity solely through the model coefficients, the OWA model is designed as:

$$
\begin{aligned}
\frac{\partial \rho R}{\partial t}+\frac{\partial \rho U_{j} R}{\partial x_{j}} & =\frac{\partial}{\partial x_{j}}\left[\left(\mu+\sigma_{R} \mu_{T}\right) \frac{\partial R}{\partial x_{j}}\right]+C_{1} \rho R S+\rho C_{2 k \omega} \frac{R}{S} \frac{\partial R}{\partial x_{j}} \frac{\partial S}{\partial x_{j}} \\
& -\rho C_{2 k \epsilon} \min \left[\left(\frac{R}{S}\right)^{2} \frac{\partial S}{\partial x_{j}} \frac{\partial S}{\partial x_{j}} ; \frac{C_{l} S R^{2}}{\nu}\right]
\end{aligned}
$$

where $C_{l}$ is defined subsequently and the eddy-viscosity $\mu_{T}=f_{\mu} \rho R$.

The physical relevance of elliptic-relaxation blending is to reinforce wall-effect, thereby suppressing the production of eddy-viscosity near a surface. To revive this phenomenon in the current model, the elliptic relaxation equation of Elkhoury [10] is invoked herein with the source term replaced by 1.0 :

$$
-L_{R}^{2} \nabla^{2} f_{R}+f_{R}=1.0
$$

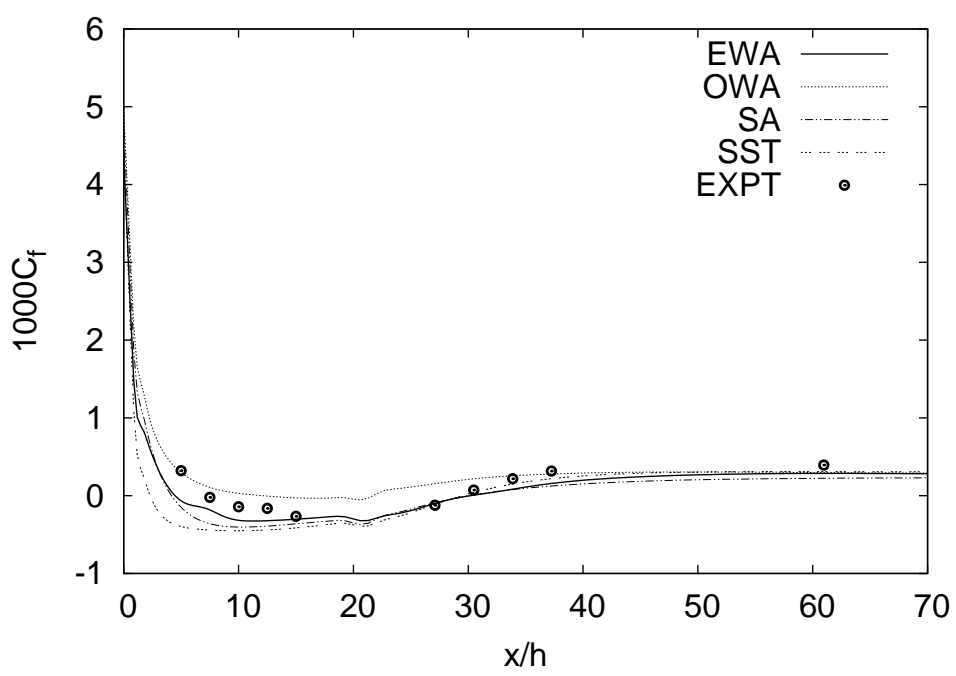

Figure 3: Skin-friction coefficient of diffuser flow along deflected bottom wall

Note-worthily, the near-wall turbulence eddies follow the Kolmogorov scaling (i.e., turbulence fluctuations depend on the laminar viscosity). Therefore, $\nu / S$ can be used for the viscous scaling, serving as a lower bound on the turbulent length scale:

$$
L_{R}^{2}=\max \left(\frac{C_{l} R}{3} ; C_{l} \nu\right) / S \approx C_{l} \nu \sqrt{1+\frac{\chi^{2}}{9}} / S
$$

where $C_{l}=4+\sqrt{\chi}$ and coefficients associated with the length scale $L_{R}$ are determined from fully-developed turbulent channel and complex (i.e., flow with separation and reattachment) flow simulations. The virtue of Eq. (6) is that unlike the Poisson equation, it requires no special numerical treatment (i.e., the Laplace operator is relatively easy to treat and is particularly easy 
with the modification $\left.-L_{R}^{2} \nabla^{2}+1\right)$. It can be easily solved in parallel with the $R$-equation with an initial guess $0 \leq f_{R} \leq 1$ everywhere except on the wall boundaries where $f_{R}=0$. It should be noted that the damping function $f_{\mu}$ is introduced to relax the viscous length-scale coefficient $C_{l}$. In other words, $f_{\mu}$ avoids the use of a larger value of $C_{l}$ in the viscous sub-layer and the wall-blocking effect is partially shared by the damping function.

It is worth mentioning that the inverse viscous scaling $S / \nu$ is used in Eq. (4) with the $C_{2 k \epsilon^{-}}$ destruction term to avoid singularity when $\frac{1}{S^{2}} \frac{\partial S}{\partial x_{j}} \frac{\partial S}{\partial x_{j}} \sim \infty$ (i.e., strain-rate $S$ reaches very small values). However, the limiter used in this work is:

$$
\frac{C_{l} S R^{2}}{\nu}>>\frac{R^{2}}{S^{2}} \frac{\partial S}{\partial x_{j}} \frac{\partial S}{\partial x_{j}}
$$

and the original formulation is eventually recovered. In addition, several numerical computations dictate that the constant coefficients given by the OWA model are too cumbersome to be implemented due to the introduction of elliptic blending. Therefore, the following parametric relations are set to the EWA model:

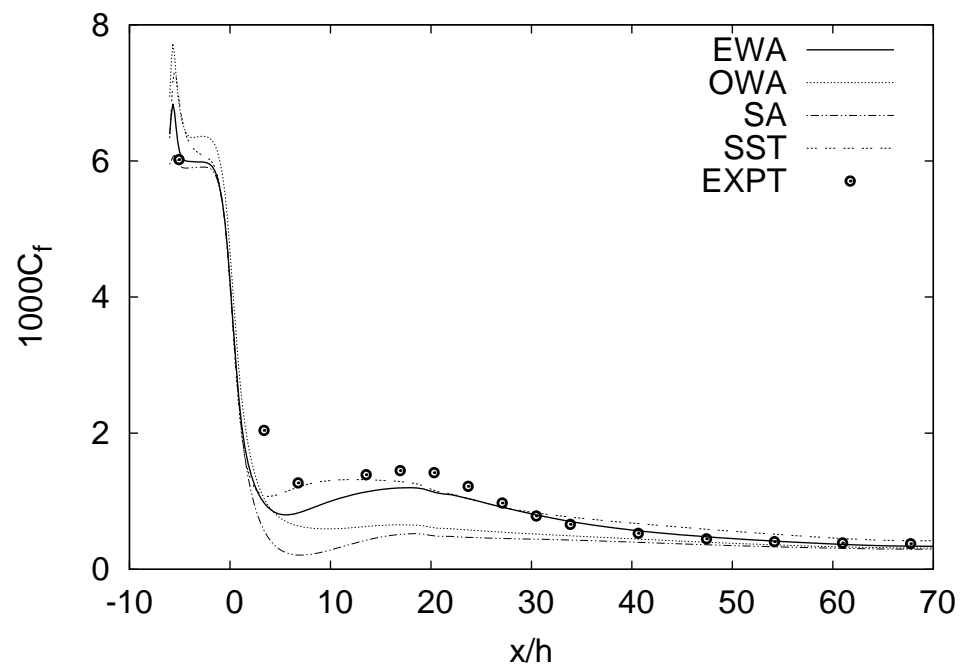

Figure 4: Skin-friction coefficient of diffuser flow along straight top wall

$$
\begin{aligned}
f_{\mu} & =\frac{\chi^{3}}{\chi^{3}+C_{w}^{3}}, & C_{1} & =f_{R}-1.0+C_{1 k \epsilon} \\
C_{2 k \omega} & =10 C_{1 k \epsilon}\left(1.0-f_{R}\right), & C_{2 k \epsilon} & =2.0-f_{R}+\min \left(A_{k \omega} ; C_{1 k \epsilon}\right) \\
A_{k \omega} & =\sqrt{\frac{|S-W|}{\max (S ; W)}}, & C_{w} & =C_{l}
\end{aligned}
$$

The revised constants are: $C_{1 k \epsilon}=0.12$ and $\sigma_{R}=0.769$. To this end, it can be stressed that the parameter $A_{k \omega}$ is activated when $S \neq W$ (i.e., non-equilibrium flows). A plot of $f_{R}$ based on Eq. (5) for a developed channel flow is shown in Fig. 1. As can be seen, the relaxation function $f_{R} \sim 1.0$ after the viscous sub-layer. Note that referring to the OWA model, the $C_{2 k \omega}$-crossdiffusion term in the EWA model is limited to be employed near solid walls to minimize the free-stream sensitivity of turbulence model. 
Remarkably, with the elliptic blending strategy, the net production $\left(f_{R}-1+C_{1 k \epsilon}\right) R S$ has been negative at near-wall (e.g., viscous) regions; however, quickly becomes positive away from the solid wall as is evidenced from Fig.1. This tendency is due to an appropriate choice of length scale associated with the relaxation equation. As mentioned earlier, the wall-blocking effect is partially shared by $f_{\mu}$ signifies that a further reduction in the production term by the elliptic relaxation is suppressed. This is an advantage of integrating both viscous (low-Reynolds number) and blocking (i.e., non-local) effects in a turbulence model, having positive implications. In all computations, $R$ has been non-negative and the proposal for elliptic blending can be thought of as an interpolation method, existing inside the wall-vicinity with the interpolation coefficient provided by a non-local model.
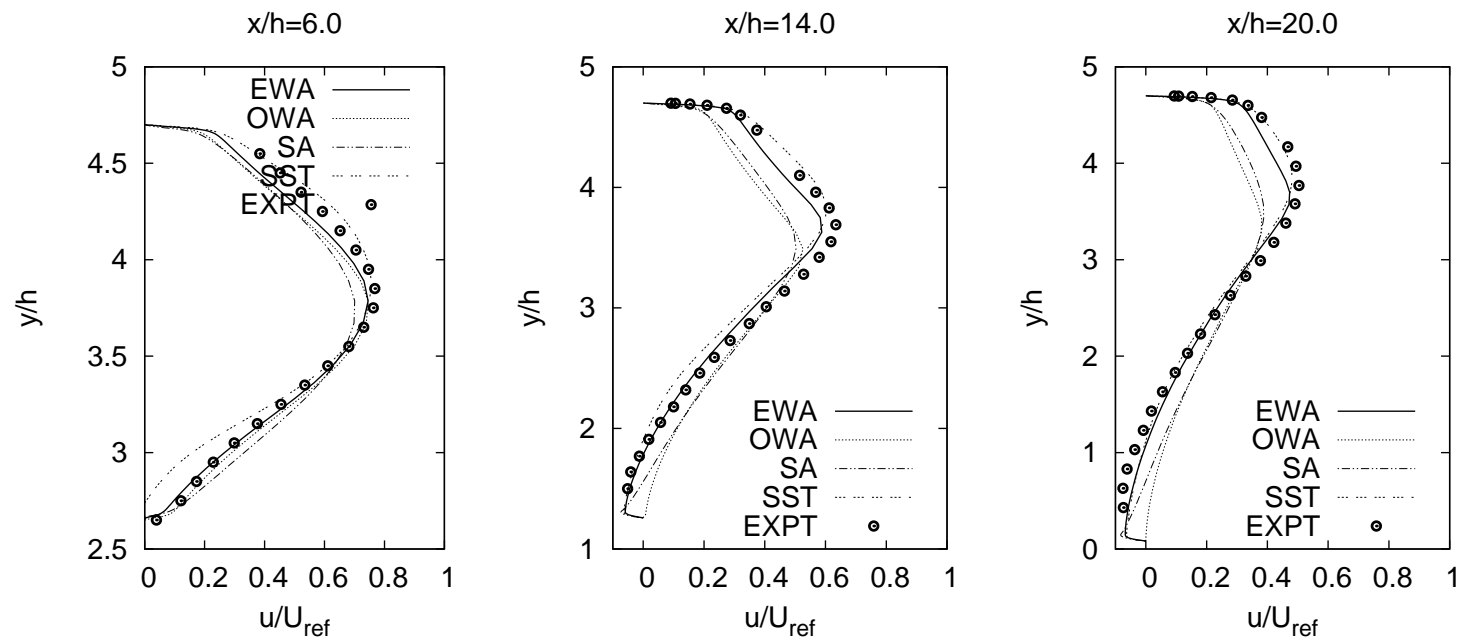

Figure 5: Mean velocity profiles at selected locations for diffuser flow

\section{Numerical Computations}

To evaluate the accuracy of proposed modifications to the OWA model, a fully developed turbulent channel flow, an asymmetric plane diffuser flow and the flow over an Onera-M6 wing are computed. To evaluate the model reliability, the current model predictions are compared with those from the widely-used SA model [13] and Menter SST (shear-stress transport) $k-\omega$ [14] model. A cell-centered finite-volume scheme combined with an artificial compressibility approach [15-18] is employed to solve the governing equations.

\section{a) Fully-Developed Turbulent Channel Flow}

The computation is carried out for a fully developed turbulent channel flow at $R e_{\tau}=395$ for which turbulence quantities are available from the DNS data [19]. The computations are conducted in the half-width $h$ of a channel, using the one-dimensional RANS solver. To ensure the accurate resolution of viscous sub-layer, the first grid node near the wall is placed at $y^{+} \approx$ 0.3. A $1 \times 64$ nonuniform grid is used across the channel half-width which is sufficient to ensure a grid-independent numerical solution. Comparisons are made by plotting the results in wall units. The results for the velocity profile shown in Fig. 2 indicate that the EWA model prediction is qualitatively good relative to the OWA model. 


\section{b) Turbulent Flow in an Asymmetric Plane Diffuser Flow}

To further evaluate the performance, the models are applied to simulate the flow in a plane asymmetric diffuser with an opening angle of $10^{\circ}$, for which measurements are available [20]. The expansion ratio of 4.7 is sufficient to produce a separation bubble on the deflected wall. This configuration provides a test case for adverse pressure driven wall-bounded separated flow. The
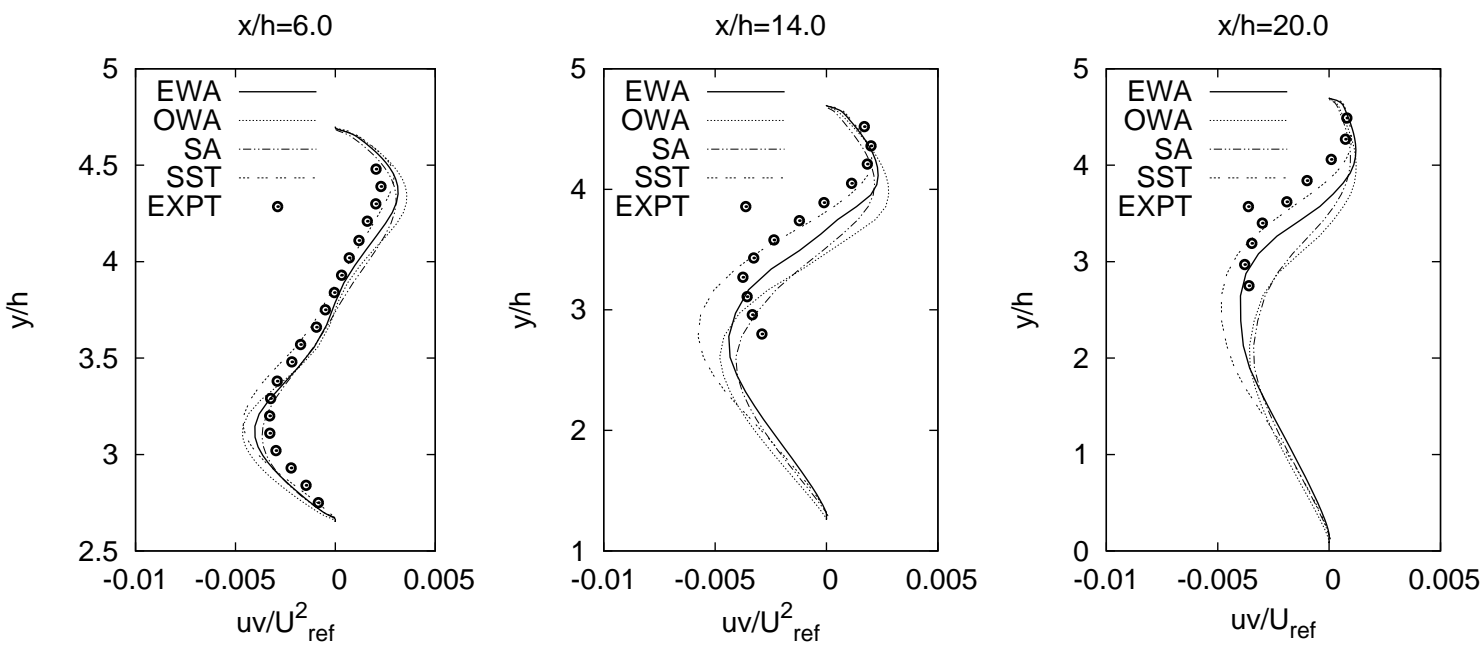

Figure 6: Shear stress profiles at selected locations for diffuser flow

entrance to the diffuser consists of a plane channel to invoke fully developed turbulent flow with $R e=20000$ based on the center-line velocity and the channel height.

Computations with a $120 \times 72$ nonuniform grid resolution are found to be accurate to obtain the grid-independent solution. The length of the computational domain is $76 h$, where $h$ is the inlet channel height. The thickness of the first cell remains below one in $y^{+}$unit on both deflected and flat walls. Figures 3 and 4 show the predicted skin-friction coefficients $C_{f}$ for all models. The proposed elliptic modification to OWA model shows improved predictions. The relatively poor prediction from the OWA model can be attributable to its wall-distance dependent blending function. Significant improvements are also observed in the mean velocity and shear profiles as shown in Figs. 5 and 6, respectively.

\section{c) Onera-M6Wing}

The Onera-M6 wing is a widely used three-dimensional test case to validate numerical methods and turbulence models for transonic flows. The flow-field is computed at a free-stream Mach number of 0.8395 , an angle of attack of $3.06^{\circ}$ and the free-stream Reynolds number of $11.71 \times$ $10^{6}$. A structured grid used in the simulation consists of four blocks with $1,572,864$ cells and the minimum normalized grid spacing to the wall is $2 \times 10^{-5}$. The main feature of this test case is recognized as the interactions of shock-wave and boundary-layer, and the separation induced by the strong shock (i.e., shock induced boundary-layer separation). However, the current study focuses on the validation of turbulence models based on only available experimental data for pressure coefficients [21].

The pressure coefficient results are compared over the wing sections located at $y / b=20$, 44, 65, 80, 90 and 95\% half-span in Fig. 7. It is observed that all models match the experiments very well. Slight over-predictions appear near the leading edge on the upper wing surface, but they are very minor. In addition, the pressures on the lower side of the wing as well as those at 
the trailing edge are well predicted and the overall profiles are captured very well by all models. The difference in the pressure distributions between the SA and other models is not distinct. Nevertheless, other computations differ slightly from those of the SA model, especially on the upper wing surface.

\section{Conclusion}

The one-equation Wray-Agarwal (WA) model is modified to replace the blending function $f_{1}$ (containing the wall-distance) by an elliptic relaxation method that accounts for the non-local characteristics of near-wall turbulence. The parameterized coefficients with the elliptic function are optimized for several simple and complex flows, three of which are presented herein. It is shown that the elliptic blending function $f_{R}$ improves the predictions of wall-bounded flows with small regions of separation. Comparing the predicted results with DNS and experimental data demonstrates that the EWA model offers improvements over the OWA and SA models and remains competitive with the SST $k-\omega$ model. In addition, the EWA model is walldistance-free (wdf) and can be easily applied to arbitrary complex computational domains with structured/unstructured grids; the wdf-feature makes it advantageous over OWA, SA and SST models.

\section{REFERENCES RÉFÉRENCES REFERENCIAS}

1. Durbin PA: Near-Wall Turbulence Closure Modeling without Damping Function. Theoretical and Computational Fluid Dynamics, 3:1-13;1991.

2. Rahman MM, Siikonen T: Low-Reynolds Number $k-\epsilon$ Model with Elliptic Relaxation Function. AIAA Journal, 44(2):392-396;2006.

3. Rahman MM and Siikonen T: A Simplified v2-f Model for Near-Wall Turbulence. International Journal for Numerical Methods in Fluids, 54:1387-1406; 2007

4. Rahman MM, Siikonen T: Near-Wall Turbulence Modeling with Elliptic Relaxation Approach. Progress in Computational Fluid Dynamics, 8:258-269;2008.

5. Rahman MM, Siikonen T: An Eddy Viscosity Model with Elliptic Relaxation Approach. International Journal of Heat and Fluid Flow, 30:319-330;2009.

6. Park TS, Sung HJ: A New Low-Reynolds Number k-Q-f $\mu$ model for Predictions Involving Multiple Surfaces. Fluid Dynamics Research, 20:97-113;1997.

7. Durbin PA, Mansour NN, Yang Z: Eddy viscosity transport model for turbulent flow. Physics of Fluids, 6(2):1007-1015;1994.

8. Rahman MM, Siikonen T, Agarwal RK: Improved Low Re-number One-Equation Turbulence Model. AIAA Journal, 49:735-747;2011.

9. Rahman MM, Wallin S, Siikonen T: Exploring $\mathrm{k}$ and $\mathrm{Q}$ with R-Equation Model Using Elliptic Relaxation Function. Flow, Turbulence and Combustion, 89:121$148 ; 2012$.

10. Elkhoury M: On the eddy viscosity transport models with elliptic relaxation. Journal of Turbulence, 18(3):240-259;2017.

11. Wray TJ and Agarwal RK: Low-Reynolds Number One-Equation Turbulence Model Based on k- $\omega$. AIAA Journal, 2015:1-112; doi:10.2514/1.J053632.

12. Wray T: Development of a One-Equation Eddy Viscosity Turbulence Model for Application to Complex Turbulent Flows. Engineering and Applied Science Theses \& Dissertations. 214. Washington Uiversity in St. Louis, USA, 2016.

13. Spalart PR, Allmaras SR: A One-Equation Turbulence Model for Aerodynamic Flows. AIAA Paper No. 92-0439, 1992. 
14. Menter FR: Two-Equation Eddy-Viscosity Turbulence Models for Engineering Applications. AIAA Journal, 32(8):1598-1605; 1994.

15. Rahman MM, Rautaheimo P, Siikonen T: Numerical Study of Turbulent Heat Transfer from a Confined Impinging Jet Using a Pseudo-Compressibility Method. 2nd International Symposium on Turbulence, Heat and Mass Transfer, Delft, The Netherlands. pp. 511-520, 1997.

16. Rahman MM, Siikonen T: An Artificial Compressibility Method for Incompressible Flows. Numerical Heat Transfer, Part B, 40:391-409;2001.

17. Rahman MM, Siikonen T: A Dual-Dissipation Scheme for Pressure-Velocity Coupling. Numerical Heat Transfer, Part B, 42:231-242;2002.

18. Rahman MM, Siikonen T: An Artificial Compressibility Method for Viscous Incompressible and Low Mach Number Flows. International Journal for Numerical Methods in Engineering, 75:1320-1340;2008.

19. Mansour NN, Kim J, Moin P: Reynolds-Stress and Dissipation-Rate Budgets in a Turbulent Channel Flow: Journal of Fluid Mechanics, 194:15-44;1988.

20. Buice C, Eaton JK: Experimental Investigation of Flow Through an Asymmetric Plane Diffuser. Technical Report, Center for Turbulence Research, Stanford University, California. pp. 94305-3030, 1997.

21. Schmitt V, Charpin F: Pressure distributions on the ONERA M6-Wing at Transonic Mach Number. in AGARD-AR-138, B1-1-B1-44, May 1979. 

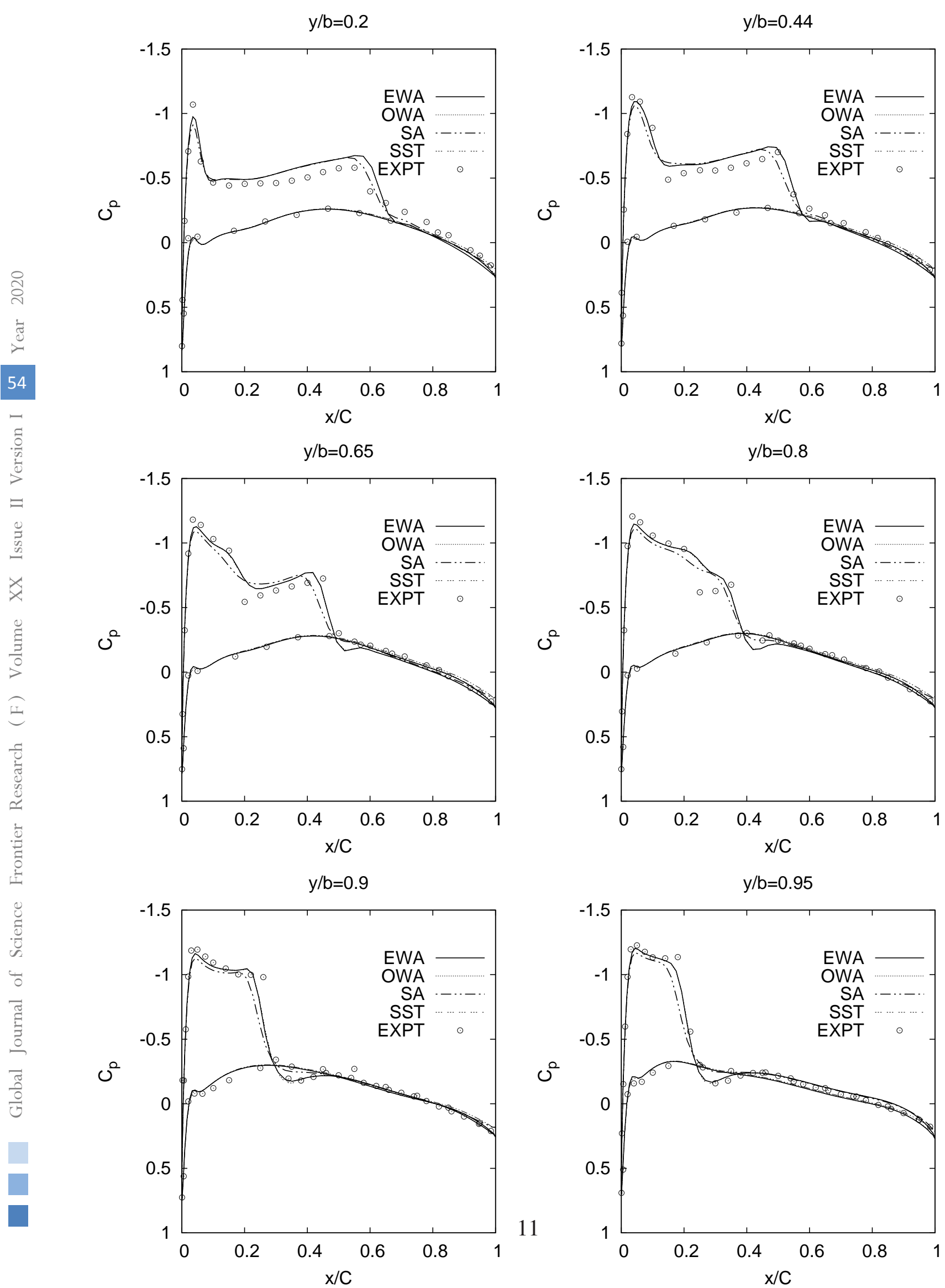

Figure 7: Wall-pressure coefficients at selected cross sections of Onera-M6 wing. 